\title{
Opt-out consent in children's emergency medicine research
}

Professor Tony Long (Corresponding author)

Professor of Child \& Family Health

School of Health \& Society

University of Salford

Salford

M6 6PU

United Kingdom

ORCID: https://orcid.org/0000-0003-2726-8798

t.long@salford.ac.uk

+44 (0) 1612952750 [Private mobile for journal use only - 07515634315 ]

Professor Andrew Rowland

Consultant in Paediatric Emergency Medicine

Emergency Department, North Manchester General Hospital

The Pennine Acute Hospitals NHS Trust, Manchester, UK

and

Professor of Paediatrics

School of Health \& Society

University of Salford

Salford

ORCID: https://orcid.org/0000-0001-9564-0032

a.rowland@salford.ac.uk

Dr Sarah Cotterill

Senior Lecturer

Centre for Biostatistics,

School of Health Sciences

University of Manchester, UK

ORCID: https://orcid.org/0000-0001-9564-0032

sarah.cotterill@manchester.ac.uk

Professor Steve Woby

Director of Operations: Research \& Innovation

Northern Care Alliance NHS Group

Salford, UK

and

Professor

School of Health \& Society

University of Salford

Salford

ORCID: https://orcid.org/0000-0002-2602-8613

steve.woby@SRFT.nhs.uk

Calvin Heal

Research Fellow

Centre for Biostatistics,

School of Health Sciences

University of Manchester, UK

ORCID: https://orcid.org/0000-0002-6445-1551

calvin.heal@manchester.ac.uk 
Natalie Garratt

Research \& Innovation Partnerships Manager

Northern Care Alliance NHS Group

Salford, UK

natalie.garratt@srft.nhs.uk

Steve Brown

Academic Network Manager

Northern Care Alliance NHS Group

Salford, UK

stephen.brown@manchester.ac.uk

Dr Damian Roland

Consultant in Paediatric Emergency Medicine

Children's Emergency Department

Leicester Royal Infirmary, Leicester, UK

and

Associate Professor in Paediatric Emergency Medicine

SAPPHIRE Group

University of Leicester

Leicester, UK

ORCID: https://orcid.org/0000-0001-9334-5144

dr98@le.ac.uk

\section{CORRESPONDING AUTHOR}

Professor Tony Long, Professor of Child \& Family Health, the University of Salford, SALFORD M6 6PU.

t.long@salford.ac.uk

+44 (0) 1612952750

\section{AUTHOR CONTRIBUTIONS}

All authors made a substantial contribution to the conception or design of the work; or to the acquisition, analysis, or interpretation of data for the work. In addition...

TL: $\quad$ Drafted the manuscript, revised it critically for important intellectual content, and approved the final version

AR: Contributed to the editing of the manuscript, revising it critically for important intellectual content, and approved the final version

SC: Contributed to the editing of the manuscript, revising it critically for important intellectual content, and approved the final version

SB: Contributed to the editing of the manuscript, approved the final version

$\mathrm{CH}$ : Contributed to the editing of the manuscript, approved the final version NG: Contributed to the editing of the manuscript, approved the final version

SB: Contributed to the editing of the manuscript, approved the final version

DR: Contributed to the editing of the manuscript, revising it critically for important intellectual content, and approved the final version 


\section{Opt-out consent in children's emergency medicine research}

\section{ABSTRACT}

There is global acceptance that individuals should be allowed to decide whether or not to take part in research studies, and to do so after being informed about the nature of the research and the risk that might attach to participation. The process of providing detailed information before seeking consent (formalised by signatures) in advance of undertaking research procedures may not be possible in some circumstances, and sometimes an amended approach may be adopted.

The use of opt-out consent has been recognised as a valid and ethical means of recruiting participants to studies particularly with large samples and where the risk to participants is small. However, it is sometimes misunderstood and can be a problematic factor in being accepted by research ethics committees and governing authorities. This may be due partly to differing expectations of the amount of information and support offered, together with the nature of the process that is adopted to ensure that a decision has been made rather than consent simply being assumed.

In accordance with ongoing discussions with young people, and following consultation with parents, an opt-out consent strategy including varied means of providing information was employed in a large study of 44,501 cases of children attending emergency or urgent care departments. The study was conducted over more than 12 months in dissimilar emergency departments and an urgent care unit, and was designed to support better decision-making in paediatric emergency departments about whether children need to be admitted to hospital or can be discharged home safely. Robust analysis of the factors that exerted the greatest impact on predicting the need to admit or the safety of discharging children led to a revised version of a an existing tool. In this article we review approaches to consent in 
research, the nature and impact of opt-out consent, the factors that made this an effective strategy for this study, but also more recent concerns which may make opt-out consent no longer acceptable.

Key words: Emergency department; Opt-out consent; Children, Ethics, Research

\section{THE BASIS OF CONSENT IN HEALTHCARE RESEARCH}

Both deontological (applying principles and rules) and consequentialist (judging actions by their consequences) approaches are adopted to ethical decision-making. Explicitly in a consequentialist approach, and practically in a deontological approach, the notion of respect for autonomy is of the greatest importance. This means that competent individuals must be allowed to make decisions for themselves on actions that will affect them significantly (as far as this is possible and with due regard to the rights of others), and they should have access to sufficient information before deciding.[1] However, even this draws disagreement.[2,3]

There is no statute or other legal basis in England, Wales or Northern Ireland for consent by children to research (rather than to treatment). For clinical trials of an investigational medicinal product it is a Health Research Authority requirement that from the age of 16 children can give consent for their own involvement but someone with parental responsibility must give consent for those under 16 years. It is often assumed that common law principles that support the right of children to provide consent to medical treatment if they demonstrate the competence to understand information and to assess the risks involved should be applied to research studies. This understanding relies partly on the quality and format of information that is provided by the researcher. Most guidance avoids any suggestion of specific age limits for children to give consent. The vague notion 
of assent rather than consent has been roundly criticised as being contradictory and disrespectful of children.[4]

The determination to avoid coercion whether deliberately or unintentionally should be applied in recruitment to research studies, the process of gaining consent being central to this. Consent is addressed in many ways for research studies: active consent, assumed consent, delayed consent, opt-out consent, and sometimes research without consent. Each is considered below. The discussion here focusses particularly on the nature and acceptability of opt-out consent in which information is provided and consent sought, but no further action is needed by the participant to indicate their agreement.

\section{ACTIVE CONSENT}

Most usually, a process of "active consent" is required in which potential participants are provided with comprehensive information about the study (usually in printed form), allowed time to think about whether or not to participate, and then invited to sign a consent form confirming agreement with a series of statements relating to the information that has been provided. Over time, issues have arisen and problems have been identified, more likely because of unforeseen or new risks though also occasionally because of poor practice, and this has the effect of adding cumulatively to the expected content of information sheets. The need for substantial statements relating to European Union General Data Protection Regulation 2016/679 (GDPR) requirements is a recent example. In England the Health Research Authority (HRA) notes that "participant information sheets are often too long and complex and their length and complexity is increasing (p5)". For a clinical trial of an investigational medicinal product (CTIMP or drug trial), a patient information sheet would normally be about 20 pages of A4 paper. However, studies with less risk to participants or with a simpler design should require far less information. HRA offers 
guidance and examples of information sheets and consent forms for researchers. It advises that "not all of the information provided in traditional, lengthy information sheets will always need to be provided to participants at the outset when initially seeking their participation" since most of the detail could be provided later if core issues of the nature, risks, possible implications and significance of participation are addressed first. [5] HRA includes an example of a short version for a low-risk drug trial which could fit on three sides of A4 paper.

Active consent is vital for many research studies, especially those carrying greater risk to participants, but imparting information with comprehensive detail yet in sufficient brevity and accessibility is a significant task. Moreover, allowing time for potential recruits to consider their decision, and often needing to seek consent again as developments in a trial force changes to the information, requires a lengthy period of engagement with participants. Fortunately, many studies which do not involve drug administration or changes in treatment will have less detail to convey and fewer risks to be considered, and so the corresponding information sheet may be much shorter; often less than four pages.

\section{RESEARCH WITHOUT CONSENT}

Securing consent from participants before involving them in a research study is not a

universal expectation. A number of situations may lead to research being conducted on human subjects either without their explicit consent or without them ever being aware of the research.

\section{Studies that might not be considered to be research}

For years NHS research ethics bodies, currently the Health Research Authority (HRA), have differentiated between research (as in the Frascati definition)[6] and both service 
evaluation and audit.[7] Research using only routinely collected, anonymised clinical data would not normally require consent, being categorised as service evaluation or audit. Such studies would not require NHS research ethics committee (REC) scrutiny. Regardless, universities and care organisations may require independent ethics and governance review, and researchers may choose to seek consent in the normal manner.

Categorisation of research to be included or excluded by the Frascati definition can be a matter of judgement and opinion.

\section{Assumed consent}

Sometimes information is provided but there is no action to confirm consent, and other times the subjects are not informed at all. Anonymised blood testing is an example of the latter. Since 1989 there have been programmes of unlinked anonymised blood testing in the US, the UK and other European states.[8] Additional or discarded body fluid samples are collected with no extra risk from specified populations for ongoing estimation of the prevalence of blood-borne diseases. In the UK and the USA this has been done without consent. Since the samples are already being taken, and no link can be made to the participant when the result is known, the benefit to public health is held to outweigh the need for consent. Elsewhere this has not been found acceptable.[8]

\section{Delayed consent}

In particular circumstances, it may be impossible to gain consent before the data is collected so the study proceeds without consent until some time later. This is known as delayed or deferred consent. Often, this will relate to emergency situations in which delaying a clinical intervention to gain consent might cause harm.[9,10] Potential participants may be unconscious or seriously ill, therefore lacking the capacity to offer consent. Even consulting others (such as parents) may not be reasonably practical, or it 
may be unfair and unreasonable to ask them to read or listen to information about a study when they are distressed. At a later time, when the situation is less stressful or the patient is able to consider participation they are approached with information about the study and asked if existing data can be retained or if they are willing to continue in the study.

\section{Necessary deception}

It may be possible to provide information but to do so would alter the responses that are to be investigated, so misleading information is provided (and consent sought on this basis) with the truth revealed only after data collection has been completed ("false consent"). $[11,12]$ Some psychology studies involve processes that could be modified by participants if they were made aware of them, so participants may be deceived about the precise nature of the research when consent is sought, the true purpose being explained to them later. A classic example is the well-known and influential study by Stockwell on "the unpopular patient".[13] Further examples and a comprehensive analysis of the use of deception in research are available.[14]

While consent may be gained for something linked to the topic to maintain the deceit, it is not specifically for what was done in the study. The researcher relies upon the benign nature of the intervention or experience and the willingness of the individual to agree to use of the data in retrospect. This is held to be appropriate only if the dignity and autonomy of the participants are protected, and where it is understood that participants would be unlikely to object or feel discomfort when the true purpose is revealed.[15]

\section{OPT-OUT OR PASSIVE CONSENT}

\section{Presumption of consent}

An alternative to active consent, without resorting to delayed or absence of consent, is inviting potential participants to opt-out of a study. A version of this was used 
internationally for many years in large medical trials;[16,17] presuming that non-response to an invitation to participate indicated acceptability of continued involvement in the study. However, reservation has been expressed about reliance on participants failing to indicate a preference rather than ensuring that they agree to participate. A brief but cogent argument has been offered about linking the degree of risk to the degree of effort expected of potential participants to indicate acceptance.[18] Increasingly, RECs and institutional review boards have demanded the adoption of active consent procedures.

\section{Non-presumptive opt-out consent}

A better-conceived strategy to opt-out consent is to provide information about the study to all potential participants and require that those who decline to participate do so by a nocost, simple, yet clear means of response. Information is provided, consent is sought, a response is required only if the potential participant chooses not to be included, and opting out is the end of involvement.

\section{The impact of opt-in and opt-out consent on sampling}

A study of angina patients in the UK found opting in to result in a biased sample and lower response than opting out.[16] This was confirmed in a US study when the opt-out recruitment rate was three times greater than the opt-in recruitment rate and partly neutralised the recruitment bias of opt-in methods (which tend to recruit more motivated patients who are not representative of the target population).[19] Opt-out consent for viral hepatitis testing was found to be more efficient in a Dublin emergency department,[20] while opt-out consent served to reverse selection bias regarding harder-to-reach groups (such as homeless people) for similar testing in a London emergency department.[21] An Australian study also found that a diverse, multi-lingual population of hospital patients 
favoured opt-out consent for secondary research with digital data, and this led to a more broadly representative sample.[22]

Irish researchers recruited 1178 participants to a cohort study of antibiotic prescribing and resistance with multiple options to opt-out (letter, telephone and website).[23] A recruitment rate of $85.5 \%$ was achieved, with only two complaints. No difference was identified between participants and those who opted out in terms of age, gender or diagnosis. In the US, researchers reported reduced sampling bias and greater recruitment from opt-out consent. They note that only those who are especially unwilling to participate (or disinterested) are likely to opt-out.[24] A further US study highlighted the need for careful consideration of context when interpreting the outcome of opt-out consent. Similar rates of recruitment to an influenza vaccination programme were found through both opt-in and opt-out consent, but the confounding factor had been a previous epidemic season causing an unusually high rate of vaccination overall.[25]

\section{How informed is informed consent?}

Opt-out methods may be beneficial to participants, too. It has been argued that opt-out consent is invalid because there is no guarantee that the information has been understood,[2] yet it may be that the usually extensive patient information sheet (PIS) used for active consent is less likely to have been read at all and even less likely, therefore, to have been understood.[26,27] HRA, too, acknowledges that "for some studies this information may often serve to confuse rather than promote genuine understanding where presented as part of an excessively lengthy information sheet.'[28] If children and young people are to be involved, this is even more important, given the high reading age of information sheets (15.88 years) found in a review of 74 studies.[29] A Danish review 
showed similar findings.[30] The reading age of one in seven adults in England is that of a child of 9-11 years.[31] A short, clearly-worded opt-out form may increase understanding.

\section{The views of parents, children and young people}

Trials have been conducted to establish parents' views and behaviour regarding opt-out consent. In an Australian study on vaccine safety surveillance, it was found that parents accepted opt-out consent, most preferring this or no consent in the case of national surveillance of this kind.[32] Canadian parents of children in a paediatric intensive care unit (PICU) were less likely to be invited and or to wish involvement in research during time of stress.[33] In the USA, researchers avoided this situation by checking with attending clinicians about parental distress and the stability of the child's condition.[34] With this safeguard, only two of 166 parents objected to opt-out consent. It was concluded that opt-out consent was acceptable and family-friendly if parents' questions could be answered. Another US study of influenza vaccination for children also found opt-out consent to be acceptable to parents with such safeguards.[35]

Children and young people themselves have clear views on consent to research. British children of 7-15 years who had experienced emergency situations felt strongly that they should be involved in decisions about their involvement in research, even if this were only after their recovery.[36] They accepted that parents need to take a lead sometimes; a facet observed generally in children's decision-making in hospital.[37] They were adamant that the clinician should ensure that the intervention would be safe.

\section{THE APPLICATION OF OPT-OUT CONSENT IN A PAEDIATRIC STUDY}

We undertook a one-year observational study in the urgent care centre or emergency department (ED) at three hospitals to refine and test the diagnostic accuracy of the 
Pennine Acute Hospitals NHS Trust Paediatric Observation Priority Score (PATPOPS)[38] to guide decision-making about admission or discharge of patients. Data was recorded on every eligible child, included routinely recorded physiological parameters as well as the personal judgement of the attending ED nurse. Specific systems were set up to record this electronically and to capture additional data from other systems (such as arrival by ambulance or not, or referral from other health professionals). Robust analysis of the factors that exerted the greatest impact on predicting the need to admit or the safety of discharging children led to a revised version of the existing score: the Paediatric Admission Guidance in the Emergency department (PAGE) tool.[39]

While the study was categorised as research because of the intention to produce generalizable findings, it did not require the consent of patients. However, our experience in research of children being willing to participate in studies (almost always declining a cooling-off period) but wishing to be consulted on any decision made about them led us to choose to inform them about the study before collecting the data. This decision was reinforced by our parent advisory group which advised us to tell families that the study was going on, but not to overstress the significance to families. While authors report considerable variability in the views of RECs and IRBs to opt-out consent,[35, 40] our experience with this study was of a supportive response.

With advice from the local clinical research network (CRN) and NHS research and innovation manager, we chose to employ opt-out consent. The PIS fitted on one side of A4 paper, explaining that research was being undertaken in the department to improve decision-making about admission and discharge, that only routinely collected data was being used, and that individual patients' care would not be affected in any way. We named staff in the department who could answer questions or discuss matters further. Two 
prominent statements emphasised that the consent form should be completed only to be excluded from the study. Posters in the departments advised of the study being conducted, staff were provided with sufficient information to address queries, and the study was promoted by the NHS trust via its website and social media. The information was reviewed for readability and amended until suitable for teenagers and adults.

The consent form was on the reverse of the information sheet, re-emphasising that this was to be completed only to have the child's data excluded from the study, and seeking only the child's name and date of attendance (to identify the case to be removed) and the parent's signature (or child's signature if they were competent to make the decision). The combined form was given to the family at the end of triage. We included every person under the age of 16 years and spent time in training departmental staff in the use of the instrument to ensure consistency in approach.

A total of 44,501 cases were included, with minimal opt-outs, reinforcing the effect on recruitment. At first, an unexpected number of opt-out forms were signed as parents signed in error to give consent to be included. Clearly, even the reduced amount of patient information was still not read accurately by some, and the expectation of opting-in is deeply ingrained. A revised sheet reduced this to a minimum, and more explicit verbal explanation accompanied the distribution of forms. An academic colleague has suggested that a "decline consent" form would be both more accurate and more understandable to parents and young people. This seems to be both sensible and practicable.

\section{REVISED GUIDANCE}

Despite the success of opt-out consent in this study and its potential benefits to recruitment and to participants' understanding, it may not be possible to employ this 
strategy again in NHS research. Responses to the introduction of a new European Union General Data Protection Regulation 2016/679 (GDPR) supplemented by the Data Protection Act 2018 in the UK have changed the status of opt-out consent in research. Although there are additional statements in GDPR relating to the use of children's personal data (prompted by increased use of social media), there is no substantive change in the legal basis for processing their data for research purposes. The intended meaning of European Directives or Regulations is clarified in a series of Recitals. The major change under GDPR that is relevant here is in Recital 32. ${ }^{1}$ This requires that for lawful personal data processing...

"Consent should be given by a clear affirmative act establishing a freely given, specific, informed and unambiguous indication of the data subject's agreement to the processing of personal data relating to him or her, such as by a written statement, including by electronic means, or an oral statement." It continues that a process must be employed that...

“...clearly indicates in this context the data subject's acceptance of the proposed processing of his or her personal data... Silence, pre-ticked boxes or inactivity should not therefore constitute consent."

The HRA and therefore the National Institute for Health Research (NIHR) concluded that consent must be ensured by explicit opt-in rather than by opt-out devices. The HRA updated its guidance in May and September 2018, removing opt-out consent as an approved strategy. The NIHR CRN, while not itself a policy-making body, would not issue ethical guidance in conflict with that of the HRA, and therefore removed opt-out consent from its own Recruitment Policy (V2.0) in September 2019. Effectively, this leaves researchers funded by the NIHR unable to provide for opt-out consent in further studies

\footnotetext{
${ }^{1}$ https://www.privacy-regulation.eu/en/recital-32-GDPR.htm
} 
(though GDPR, HRA and NIHR continue to accept research without consent in some circumstances). Many large studies have relied upon opt-out consent to ensure adequate sampling. Moving to opt-in consent may damage both the size of the sample and its representative nature, as discussed earlier.

\section{CONCLUDING ISSUES}

It would have been possible to conduct our NIHR-funded study without seeking NHS REC consent at all (and approval was also secured from a university REC). We chose to respond positively to the expressed preferences of parents and children since to do otherwise would be worse than not having asked at all. The additional trouble of constructing a suitable combined patient information sheet and opt-out consent form, of bringing this to the attention of every family attending the departments, and then taking further steps to correct misunderstanding of the meaning of signing the form all seemed worthwhile to us. In this case, opt-out consent allowed for brevity, provided three simple means of choosing not to have the data used in the study (immediately or later), and proved effective both in capturing the small number of cases in which consent was declined and also in allowing for large-scale recruitment of informed participants. Whatever the formal requirement, we felt morally obliged to seek consent in the reasonable format that was demanded by parents and children.

The second issue relates to the assumed bivariate nature of actively opting-in or absence of choice (silence or inactivity). This may be too simplistic. Certainly, silence or inactivity did not apply to this study. Parents of all children attending the departments received the combined form at the end of triage with a verbal explanation. Posters explained about the active study in that area, named staff were available to explain further and to answer questions, and the study had been promoted by the internet and social media. These 
actions may go further in informing and interacting than studies in which active consent is sought by post or online. We gave clear messages that the data would be collected routinely for clinical purposes regardless, that care and treatment would not change, and that there was no need to sign the form unless parents objected to the use of the data for the research study. We advised them to retain the PIS in any case. This seems to us to be much more than a pre-ticked box, silence or inactivity.

Sometimes, consent should be sought even if regulation does not require it, and the outcome of participants making an informed decision may depend on less extensive, more readily digestible information when risks are low and non-complex. Opt-out consent does not have to mean absence of information and choice, nor must it mean lack of effort to ensure understanding before deciding to participate. Its use particularly to ensure largescale recruitment should be reviewed.

\section{ETHICS}

Formal ethics approval for the study was secured: NHS REC reference 17/WM/0436, University of Salford REC reference HSR1617-175.

\section{FUNDING}

This study was funded by the National Institute for Health Research (NIHR) Research for Patient Benefit (ref PB PG 0815 20034). It used data provided by patients and collected by the NHS as part of their care and support. The views expressed are those of the authors and not necessarily those of the NIHR or the Department of Health and Social Care.

\section{CONFLICTS OF INTEREST}

None of the authors declares any conflict of interest. 


\section{REFERENCES}

1 Long T. How are these issues to be resolved? In: Long T, Johnson M, eds. Research ethics in the real world: issues for health and social care. Edinburgh: Elsevier 2007:pp 6384.

2 MacKay D. Opt-out and consent. Journal of Medical Ethics 2015;41:832-835. doi:10.1136/medethics-2015-102775.

3 Saunders B. Understanding (and) consent: a response to MacKay. Journal of Medical Ethics 2016;42:203-4. doi:10.1136/medethics-2011-100039.

4 Alderson P. Children's consent and 'assent' to healthcare research. In Freeman M, ed. Current Legal Issues Volume 14: Law and Childhood Studies. Oxford: Oxford University Press 2012:pp 174-189.

5 Health Research Authority (2019) Informing participants and seeking consent. https://www.hra.nhs.uk/planning-and-improving-research/best-practice/informingparticipants-and-seeking-consent/

6 Organisation for Economic Co-operation and Development. Frascati Manual. Guidelines for collection and reporting data on research and experimental development. 2018 http://www.oecd.org/sti/inno/frascati-manual.htm

7 Health Research Authority (2018) Is my project research? Defining research. http://www.hra-decisiontools.org.uk/research/docs/DefiningResearchTable_Oct2017-1.pdf

8 Kessel AS, Datta J, Wellings K, Perman S. The ethics of unlinked anonymous testing of blood: views from in-depth interviews with key informants in four countries. BMJ Open 2012;2:e001427. doi:10.1136/bmjopen-2012- 001427

9 Waterfield T, Lyttle MD, Shields M On behalf of Paediatric Emergency Research in the UK and Ireland (PERUKI), et al. Parents' and clinicians' views on conducting paediatric diagnostic test accuracy studies without prior informed consent: qualitative insight from the Petechiae in Children study (PiC). Arch Dis Child 2019;104:979-983.

doi:10.1136/archdischild-2019-317117

10 Woolfall K, Roper L, Humphreys A, et al. Enhancing practitioners' confidence in recruitment and consent in the EcLiPSE trial: a mixed-method evaluation of site training a Paediatric Emergency Research in the United Kingdom and Ireland (PERUKI) study. Trials 2019;20:181.

11 Wilson AT. Counterfactual Consent and the Use of Deception in Research. Bioethics. 2015;29(7):470-477. doi:10.1111/bioe.12142

12 Long T. What are the ethical issues in research? In Long T, Johnson M, eds. Research ethics in the real world: issues for health and social care. Edinburgh: Elsevier 2007:pp 4762.

13 Stockwell F. The unpopular patient. Croom helm: London 1972, 1984. 
14 McCambridge J, Kypri K, Bendtsen P, Porter J. The use of deception in public health behavioral intervention trials: a case study of three online alcohol trials. Am J Bioeth. 2013;13(11):39-47. doi:10.1080/15265161.2013.839751]

15 British Psychological Society. Code of Human Research Ethics. Leicester: BPS UK 2014. https://www.bps.org.uk/sites/bps.org.uk/files/Policy\%20-

\%20Files/BPS\%20Code\%20of\%20Human\%20Research\%20Ethics.pdf

16 Junghans $C$, Feder G, Hemingway $H$, Timmis A, Jones M. Recruiting patients to medical research: double blind randomised trial of "opt-in" versus "opt-out" strategies. BMJ 2005;331:940. doi:10.1136/bmj.38583.625613.AE.

17 Junghans C, Jones M. Consent bias in research: how to avoid it. Heart 2007;93:10245. doi:10.1136/hrt.2007.120113

18 Sommerville A. What's wrong with opting out? (Commentary) BMJ 2012;322:1220.

19 Aysola J, Tahirovic E, Troxel AB, Asch DA, Gangemi K, Hodlofski AT, Zhu J, Volpp K. A randomized controlled trial of opt-in versus opt-out enrolment into a diabetes behavioral intervention. Am J Health Promot 2016;32:745-752. doi:10.1177\%2F0890117116671673

20 O'Connell S, Lillis D, Cotter A, O'Dea S, Tuite H, Fleming C, Dalby L, Barry H, Shields D, Norris S, Plunkett PK, Bergin C. Opt-out panel testing for HIV, hepatitis B and hepatitis C in an urban emergency department: a pilot study. PLoS One 2016;11:e0150546. doi:10.1371/journal.pone.0150546

21 Evans H, Balasegaram S, Douthwaite S, et al. An innovative approach to increase viral hepatitis diagnoses and linkage to care using opt-out testing and an integrated care pathway in a London emergency department. PLOS ONE 2018;13:e0198520. doi:10.1371/journal.pone.0198520

22 Boulos D, Morand E, Foo M, et al. Acceptability of opt-out consent in a hospital patient population. Intern Med J 2018;48:84-87. doi:10.1111/imj.13666

23 Vellinga A, Cormican M, Hanahoe B, et al. Opt-out as an acceptable method of obtaining consent in medical research: a short report. BMC Med Res Methodol 2011;11:40. http://www.biomedcentral.com/1471-2288/11/40.

24 Miller CJ, Burgess JF, Fischer EP, et al. Practical application of opt-out recruitment methods in two health services research studies. BMC Med Res Methodol 2017;17:57. doi:10.1186/s12874-017-0333-5.

25 Wooton SH, Blackwell SC, Saade G, et al. Randomized quality improvement trial of opting-in versus opting-out to increase influenza vaccination rates during pregnancy. AJP Reports 2018;8:e161-e167. doi:10.1055/s-0038-1668566.

26 Ennis L, Wykes T. Sense and readability: participant information sheets for research studies. Br J Psychiatry. 2016;208:189-94. doi: 10.1192/bjp.bp.114.156687.

27 Sharp SM. Consent documents for oncology trials: does anybody read these things? Am J Clin Oncol 2004;27:570-5. doi:10.1097/01.coc.0000135925.83221.b3. 
28 Health Research Authority (2019) Applying a proportionate approach to the process of seeking consent.

https://www.hra.nhs.uk/documents/1691/Proportionate_approach_to_seeking_consent_H RA_Guidance.pdf

29 Franck L, Winter I. Research participant information sheets are difficult to read. Bull Med Ethics 2004;195:13-6.

30 Grootens-Wiegers P, De Vries MC, Vossen TE, et al. Readability and visuals in medical research information forms for children and adolescents. Science Communication 2014;37:89-117. doi:10.1177/1075547014558942

31 UK Government. 2011 Skills for life survey: a survey of literacy, numeracy and ICT levels in England. 2012. https://www.gov.uk/government/publications/2011-skills-for-life-survey

32 Berry JG, Ryan P, Duszynski KM, et al. Parent perspectives on consent for the linkage of data to evaluate vaccine safety: a randomised trial of opt-in and opt-out consent. Clinical Trials 2013;10:483-94. doi:10.1177/1740774513480568.

33 Menon K, Ward R, Canadian Critical Care Trials Group. A study of consent for participation in a non-therapeutic study in the pediatric intensive care population. Journal of Medical Ethics 2014;40:123-126. doi:10.1136/medethics-2012-101075

34 Fernandes DM, Roland AP, Morris MC. Parental opinions regarding an opt-out consent process for inpatient pediatric prospective observational research in the US. Pragmat Obs Res 2017;8:1-8. doi:10.2147/POR.S126509

35 Higgerson RA, Olsho LEW, Christie LM, et al. Variability in IRBs regarding parental acceptance of passive consent. Pediatrics 2014;134:e496-e503.

www.pediatrics.org/cgi/doi/10.1542/peds.2013-4190

36 Roper L, Sherratt FC, Young B, et al. Children's views on research without prior consent in emergency situations: a UK qualitative study. BMJ Open 2018;8:e022894. doi:10.1136/ bmjopen-2018-022894

37 Livesley J, Long T. Children's experiences as hospital in-patients: voice, competence and work. Messages for nursing from a critical ethnographic study. Int J Nurs Stud 2012;50:1242-1303. doi:10.1016/j.jinurstu.2012.12.005

38 Cotterill S, Rowland AG, Kelly J, Lees H, Kamara M. Diagnostic accuracy of PATPOPS and ManChEWS for admissions of children from the emergency department. Emerg Med J 2016;33(11):756-62

39 Heal C, Cotterill S, Rowland AG, Garratt N, Long T, Brown S, O'Connor G, Rishton C, Woby S, Roland D. Inter-rater reliability of paediatric emergency assessment: physiological and clinical features. Arch Dis Child 2020 [In press]

40 Gale C, Hyde MJ, Modi N. (2017) Research ethics committee decision-making in relation to an efficient neonatal trial. Arch Dis Child Fetal Neonatal Ed 2017;102:F291F298. https://fn.bmj.com/content/fetalneonatal/102/4/F291.full.pdf 


\section{WHAT IS KNOWN ALREADY}

It has been accepted since the Declaration of Helsinki that all humans have the right to self-determination in the decision to take part in research, and that the researcher's prime duty is to participants' welfare, taking precedence over the need for research. Consent should still be sought whenever possible from physical or intellectually vulnerable individuals. Most often in health research, opt-in consent is required, following consideration by potential participants of extensive, detailed printed information.

\section{WHAT THIS STUDY ADDS}

How participant consent is ensured can vary according to the type of data, design of the study, and risks to participants. In low-risk studies with no change to patients' treatment it has been possible to consent by declining to sign an opt-out form. UK health research authorities have rejected this for conflicting with GDPR2016/679. This interpretation may be too narrow and not in the best interests of participants or the generation of large-scale research data. 